\title{
One-pot size-controlled growth of graphene-encapsulated germanium nanocrystals
}

DOI:

10.1016/j.apsusc.2018.01.165

\section{Document Version}

Accepted author manuscript

Link to publication record in Manchester Research Explorer

\section{Citation for published version (APA):}

Lee, J. H., Lee, E. K., Kang, S. G., Jung, S. H., Son, S. K., Nam, W. H., Kim, T. H., Choi, B. L., \& Whang, D. (2018). One-pot size-controlled growth of graphene-encapsulated germanium nanocrystals. Applied Surface Science, 440, 553-559. https://doi.org/10.1016/j.apsusc.2018.01.165

\section{Published in:}

Applied Surface Science

\section{Citing this paper}

Please note that where the full-text provided on Manchester Research Explorer is the Author Accepted Manuscript or Proof version this may differ from the final Published version. If citing, it is advised that you check and use the publisher's definitive version.

\section{General rights}

Copyright and moral rights for the publications made accessible in the Research Explorer are retained by the authors and/or other copyright owners and it is a condition of accessing publications that users recognise and abide by the legal requirements associated with these rights.

\section{Takedown policy}

If you believe that this document breaches copyright please refer to the University of Manchester's Takedown Procedures [http://man.ac.uk/04Y6Bo] or contact uml.scholarlycommunications@manchester.ac.uk providing relevant details, so we can investigate your claim.

\section{OPEN ACCESS}




\section{One-pot size-controlled growth of graphene-encapsulated germanium nanocrystals}

Jae-Hyun Lee, ${ }^{1, a)}$ Eun-Kyung Lee, ${ }^{2, a)}$ Seog-Gyun Kang, ${ }^{3}$ Su-Ho Jung, ${ }^{3}$ Seok-Kyun Son, ${ }^{4}$ Woo Hyun Nam, ${ }^{5}$ Tae-Hoon Kim, ${ }^{6}$ Byong Lyong Choi, ${ }^{2, *}$ and Dongmok Whang ${ }^{3, *}$

${ }^{1}$ Department of Energy Systems Research and Department of Materials Science and Engineering, Ajou University, Suwon 16499, South Korea

${ }^{2}$ Samsung Advanced Institute of Technology, Suwon, 16674, South Korea

${ }^{3}$ School of Advanced Materials Science and Engineering, Sungkyunkwan University, Suwon, 16419, South Korea

${ }^{4}$ National Graphene Institute, University of Manchester, Manchester, M13 9PL, United Kingdom

${ }^{5}$ Center for Nanomaterials and Chemical Reactions, Institute for Basic Science (IBS), Daejeon, 34141, South Korea

${ }^{6}$ Ames Laboratory, Ames, IA 50011, United States

\footnotetext{
* Corresponding author. Tel.: +82 31 2997399; Fax: +82 312907410

E-mail addresses: blchoi@samsung.com (B.L. Choi); dwhang@skku.edu (D.Whang)

a) Jae-Hyun Lee and Eun-Kyung Lee contributed equally to this work.
} 


\section{Abstract}

To realize graphene-encapsulated semiconductor nanocrystals (NCs), an additional graphene coating process, which causes shape destruction and chemical contamination, has so far been inevitable. We report herein one-pot growth of uniform graphene-germanium core-shell nanocrystals (Ge@G NCs) in gram scale by the addition of methane as a carbon source during the thermal pyrolysis of germane. The methane plays a critical role in the growth of the graphene shell, as well as in the determination of the nucleation density and diameter of the NCs, similar to a surfactant in the liquid-phase growth of monodisperse NCs. By adjusting the gas ratio of precursors, a mixture of germane and methane, we can control the size of the Ge@G NCs in the range of $\sim 5 \mathrm{~nm}$ to $\sim 180 \mathrm{~nm}$. The Ge@G NCs were characterized by various microscopic and spectroscopic tools, which indicated that the Ge core is single crystalline, and is completely covered by the graphene shell. We further investigated the merits of the graphene shell, which can enhance the electrical conductivity of nanocrystalline materials.

Keywords: germanium, graphene, core-shell, nanocrystal, chemical vapor deposition (CVD), thermoelectric

\section{Introduction}

The incorporation of graphene with a semiconductor have been intensively studied over the past decade, because the superb physical and unique chemical properties of graphene generate synergistic effects in various areas [1-5]. For example, new types of transistors and high-speed optical modulators for next generation electronics have been invented by the integration of graphene with typical Si-based complementary metal oxide semiconductor (CMOS) technology [3, 4]. These convergence systems are particularly required for nanocrystals (NCs) research to surmount the NCs weaknesses, such as their instability, low current density, and poor reliability [6-11]. Wrapping NCs with graphene can be considered an effective route, because the $s p^{2}$-hybridized carbon network improves their physical and 
chemical properties. Encapsulation of NCs in a graphene cage is used for anode materials in the lithium-ion battery, to achieve long-life cycles and high capacity $[6,7]$. In addition, the graphene shell improved the electrical conductivity, as well as the chemical stability of the NCs for conductive ink [8]. In either case, an additional graphene coating process to realize graphene-covered NCs cannot be avoided. Chemical vapor deposition (CVD) is a widely used method for the direct growth of a graphene shell on the surface of NCs [6-8]. However, after the synthetic process, the shape of the NCs becomes irregular and the size gets polydisperse, owing to the melting point depression effects at the high graphene growth temperature. Alternatively, solution-based approaches have been reported; however, they also suffer from low crystallinity and chemical contamination issues $[12,13]$. Therefore, these approaches are not suitable for electronic devices that require high crystallinity, narrow size distribution, and a clean interface. Recently, Wang et al. and Sengar et al. introduced one-step growth methods for metal-graphene core shell NCs using organometallic precursor $[14,15]$. Although these techniques can satisfy the aforementioned requirements, they are so far only available for metal NCs.

The germanium (Ge) NCs, the group IV semiconductor, have been extensively explored for various types of electronic applications, such as transistor, battery, thermoelectric, and photovoltaics [16-19]. In particular, compared to other semiconductor materials, Ge shows a good catalytic activity for the $s p^{2}$ hybridization of carbon atoms [20-22]. In this paper, taking advantage of catalytic behavior of Ge, we demonstrate one-pot growth of graphene-coated uniform Ge NCs (Ge@G NCs) in gram scale via the conventional CVD method. The diameter of the Ge@G NCs is controlled by the precursor ratio of methane and germane. Microscopic and spectroscopic analysis confirmed that the Ge core is single crystalline, and is completely covered by the graphene shell. In addition, based on the experimental results, 
we describe the growth mechanism of the Ge@G NCs; the role of methane is critical for determination of the density and diameter of the NCs.

\section{Experimental}

\subsection{Characterization.}

The morphology and size of the Ge@G NCs were measured by JEOL JSM-7401F field emission scanning electron microscopy, operating with an accelerating voltage of $10 \mathrm{kV}$ (FESEM). The crystallinity and composition of the NCs were characterized by JEOL JEM-2100F field emission transmission electron microscopy (FE-TEM). For TEM measurement, the Ge@G NCs were suspended in ethanol, dispersed onto a $\mathrm{Cu}$ grid with a thin lacey carbon support film, and imaged at an accelerating voltage of $200 \mathrm{kV}$. Powder X-ray diffraction (XRD) measurements were performed on a D8 ADVANCE (Bruker) using $\mathrm{Cu} \mathrm{K} \alpha$ radiation $\left(\mathrm{k}=1.5406 \AA\right.$ ), and a step size of $0.04^{\circ}$. X-ray photo-electron spectroscopy (XPS) analysis was carried out by ESCA2000 spectrometry using monochromatic Al Ka radiation (1,468.6 $\mathrm{eV}$ ). The peak energies were calibrated to the $\mathrm{C} 1 \mathrm{~s}$ peak at $284.6 \mathrm{eV}$. Raman spectroscopy (Renishaw, RM-1000 Invia) with excitation energy of $2.41 \mathrm{eV}(514 \mathrm{~nm}$; Ar ion laser) was used to characterize NCs. For both XPS and Raman measurement, the Ge@G NCs were dispersed on thin gold film $(50 \mathrm{~nm})$ deposited Si substrates.

\subsection{Plasma Spark Sintering.}

The Ge@G NCs were compacted into disk-shaped pellets with a diameter of $10 \mathrm{~mm}$ and a thickness of $3 \mathrm{~mm}$ using a spark plasma sintering (SPS) technique. Table S1 shows the SPS process conditions. The density $\left(\rho_{s}\right)$ of the synthesized sample was calculated as $5.06 \sim 5.26$ $\mathrm{g} / \mathrm{cm}^{3}$, which is more than $95 \%$ of the theoretical value of Ge.

\subsection{Thermoelectric measurement.}


The electrical conductivity $(\sigma)$ was measured at $300 \sim 860 \mathrm{~K}$ using the ULVAC ZEM-3 equipment. The thermal conductivity $(\kappa)$ was calculated using the equation $\kappa_{\text {tot }}=\rho_{s} C_{p} \lambda$. The heat capacity $\left(C_{p}\right)$ value used in the equation is the $C_{p}$ value $(0.31 \mathrm{~J} / \mathrm{gK})$ of pure Ge at $300 \mathrm{~K}$ as the constant value [23]. Thermal diffusivity $(\lambda)$ values were measured under vacuum by a laser flash method (TC-9000; ULVAC, Japan).

\section{Results and Discussion}

\subsection{Growth and Characterization of the Ge@G NCs}
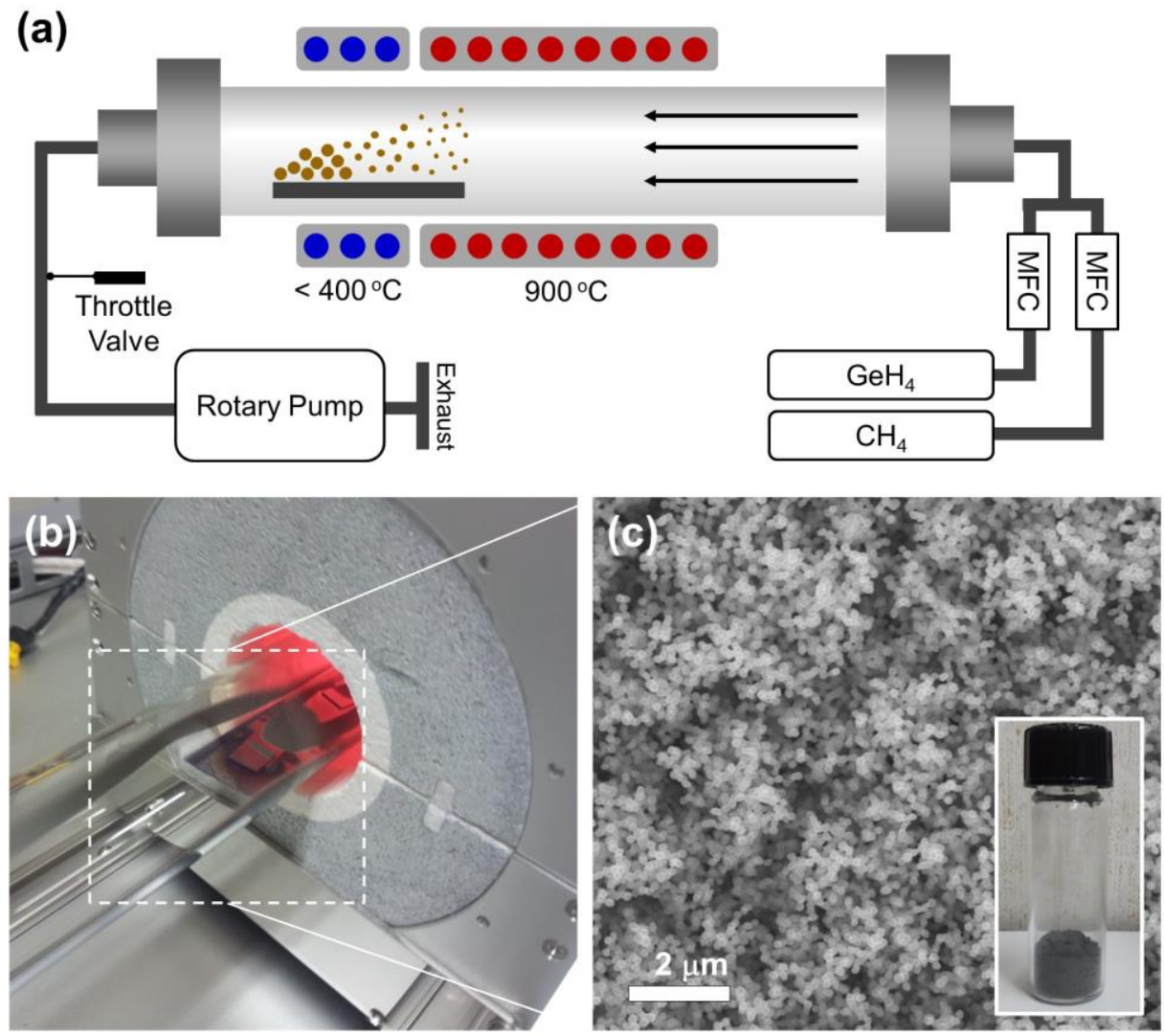

Fig. 1. (a) Schematic of the one-pot growth of the Ge@G NCs. (b) Photograph-, and (c) SEMimage of a large density of the Ge@G NCs on the collecting substrate. The inset in Fig. 1 (c) shows a photograph-image of 80 mg of the Ge@G NCs in a vial.

Fig. 1 (a) shows the schematic of the growth of Ge/graphene core/shell nanocrystals, which was demonstrated by conventional hot-wall chemical vapor deposition (CVD). Firstly, the holder for collecting the Ge@G NCs was loaded into the collection region in the quartz 
tube $(\varnothing=34 \mathrm{~mm})$, and then the chamber was evacuated to $\sim 3$ mTorr by rotary pump. Subsequently, the furnace was heated to $900{ }^{\circ} \mathrm{C}$ under Ar atmosphere (heating rate was kept at $20{ }^{\circ} \mathrm{C}$ per min). When the furnace reached the target temperature, $\mathrm{GeH}_{4}(99.999 \%$, diluted to $10 \%$ with $\left.\mathrm{H}_{2}\right)$ and $\mathrm{CH}_{4}(99.999 \%)$ as the $\mathrm{Ge}$ and carbon source, respectively, were introduced into the quartz tube, and the total pressure was maintained at 100 Torr for 120 min. All the gases were completely mixed before entering the processing zone. To avoid the aggregation of the Ge@G NCs after the growth process, the cooling block was set at the edge of the furnace, maintaining the temperature of the collecting region lower than $400{ }^{\circ} \mathrm{C}$ (see Fig. S1). Figs. 1 (b) and (c) show the photograph and SEM images of the Ge@G NCs at the cooling region during the growth process. Note that we could obtain uniform Ge@G NCs in gram scale at low methane-to-germane gas ratio (inset of Fig. 1 (c)).
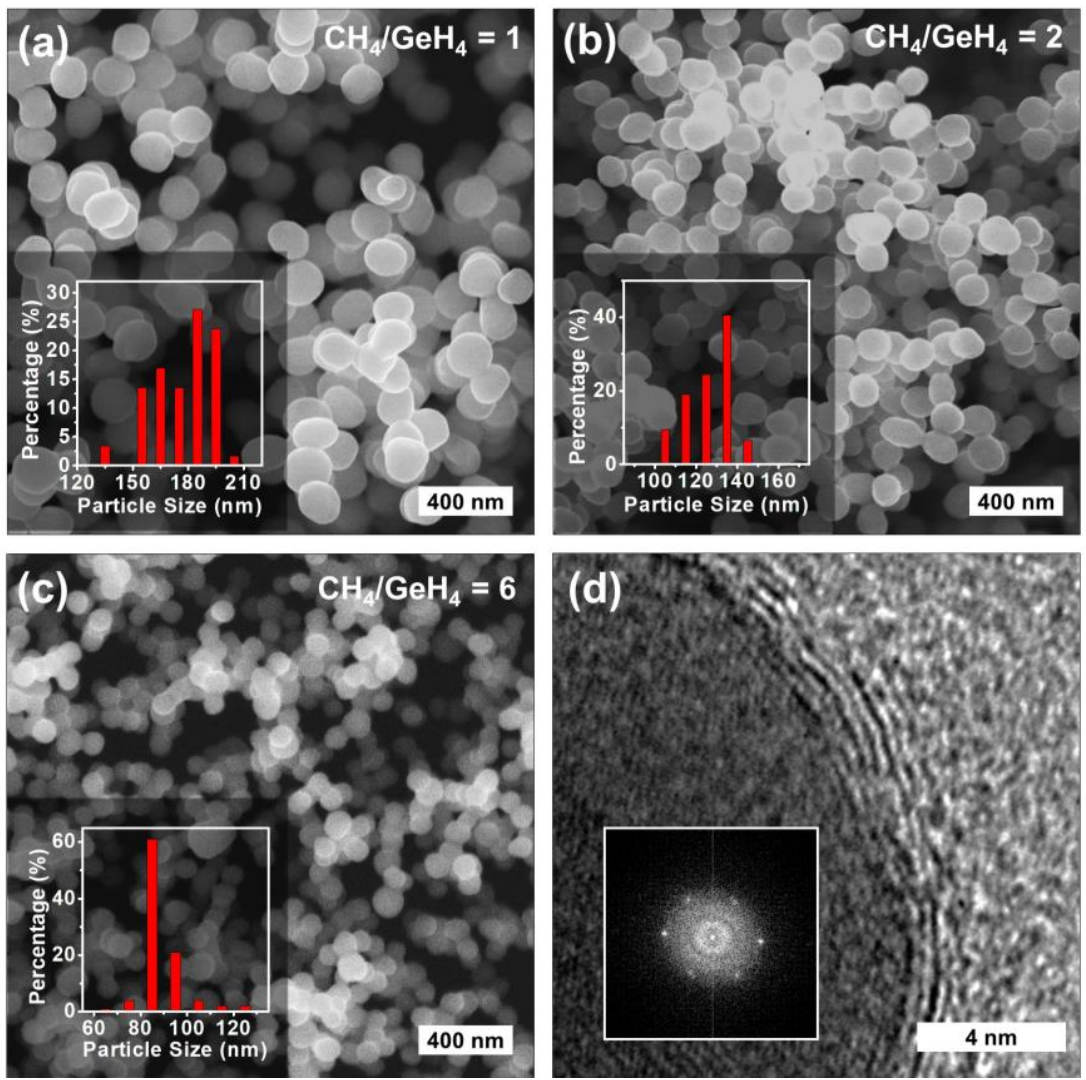

Fig. 2. (a)-(c) SEM images of the Ge@G NCs with different methane-to-germane gas ratio $\left(\mathrm{CH}_{4} / \mathrm{GeH}_{4}\right)$. Insets show the size distribution of the Ge@G NCs. (d) HR-TEM image of the Ge@G NCs. Inset shows the fast Fourier transform pattern from the Ge core. The average number of graphene layer is around 3-5. 
By changing the partial pressure of methane, it is possible to control the diameters of the Ge@G NCs (Figs. 2 (a)-(c)). As he gas ratio of methane and germane increases to 6, the average diameter of the NCs decreases from $177.7 \pm 17.3$ to $91.4 \pm 10.8 \mathrm{~nm}$. As the gas ratio is further increased to $10, \mathrm{NCs}$ as small as $5.7 \pm 1.5 \mathrm{~nm}$ are obtained (Fig. S2). Fig. S2 shows the low- and high-magnification TEM images of the mono-disperse Ge@G NCs. A detailed growth mechanism is discussed in the later section. The high-resolution TEM image of the as-grown Ge@G NCs indicates that the Ge core is single crystalline, and is completely covered by a multi-layered graphene shell (Fig. 2 (d)).
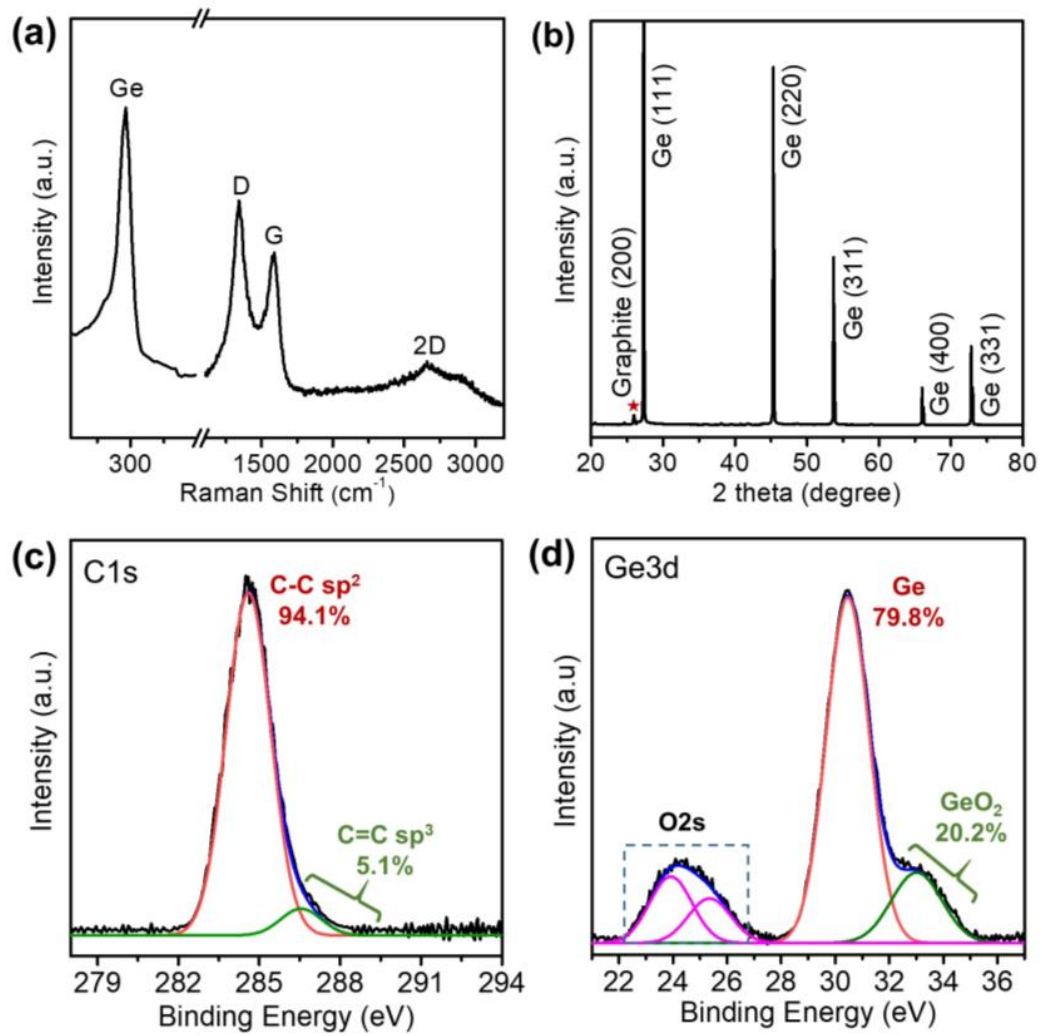

Fig. 3. (a) Raman spectra of the Ge@G NCs. (b) Powder XRD pattern of the Ge@G NCs. The red star indicates the peak from the graphene shell. (c) and (d) XPS spectra of the Ge@G NCs for the core level of $\mathrm{C} 1 \mathrm{~s}$ and the Ge $3 \mathrm{~d}$ region.

Various spectroscopic measurements were performed to verify the crystal structure and chemical properties of the Ge@G NCs. The Raman spectra in Fig. 3 (a) provides information about the crystallinity of both the Ge core and graphene shell $[24,25]$. The strong and narrow peak at around $300 \mathrm{~cm}^{-1}$, corresponding to the first order Raman signaling of Ge, indicates 
the high crystallinity of the Ge core [24]. Other obvious three peaks located at $1,350,1,580$, and $2,700 \mathrm{~cm}^{-1}$ are consistent with the representative Raman spectra of graphene, which are assigned to D band, G band, and 2D band, respectively [25]. The D band, implying the disorder of the $s p^{2}$ carbon network, might be caused by the step edges of the several-layered graphene shell or the finite particle-size effect $[8,14,25,26]$. Size of graphene domain is obtained from the integrated intensity ratio of $\mathrm{D}$ band and $\mathrm{G}$ band, indicating it is approximately $11.31 \mathrm{~nm}$ (Fig. S3) [27]. The crystal structure of the Ge@G NCs was investigated by XRD. The (111), (220), (311), and (331) planes of the diamond cubic Ge (JCPDS 4-0545), corresponding to 2 theta values of $27^{\circ}, 45^{\circ}, 55^{\circ}$, and $72^{\circ}$, respectively, are labeled in Fig. 3 (b) [16]. In particular, the peak at around $24^{\circ}$ represents the graphite $(002)$ plane (JCPDS 1-0646), supporting the existence of a multi-layered graphene shell [10].

The chemical states of the Ge@G NCs were characterized by XPS. Figs. 3 (c) and (d) show the core level peaks of C 1s and Ge $3 \mathrm{~d}$ in the XPS spectrum. The area under the peak at $284.6 \mathrm{eV}$ corresponding to the $\mathrm{C}-\mathrm{C}$ component is $94.1 \%$, whereas that of the peak at 288.8 $\mathrm{eV}$ corresponding to $\mathrm{C}=\mathrm{C}$ carbon is $5.1 \%$, confirming that the graphene shell is predominantly composed of a $s p^{2}$-hybridized carbon network [28]. However, although graphene covered the surface of the Ge core, a small portion of Ge-O peaks located at around $33 \mathrm{eV}$ is also observed [29]. These oxidation states of the Ge core can be explained by the existence of defects in the outer graphene shell $[8,30,31]$. In either case, there are no peaks related to the Ge-C alloy, because the carbon solid solubility in Ge is negligible at the growth temperature used $[20,29,32]$.

\subsection{Growth mechanism of the Ge@G NCs}




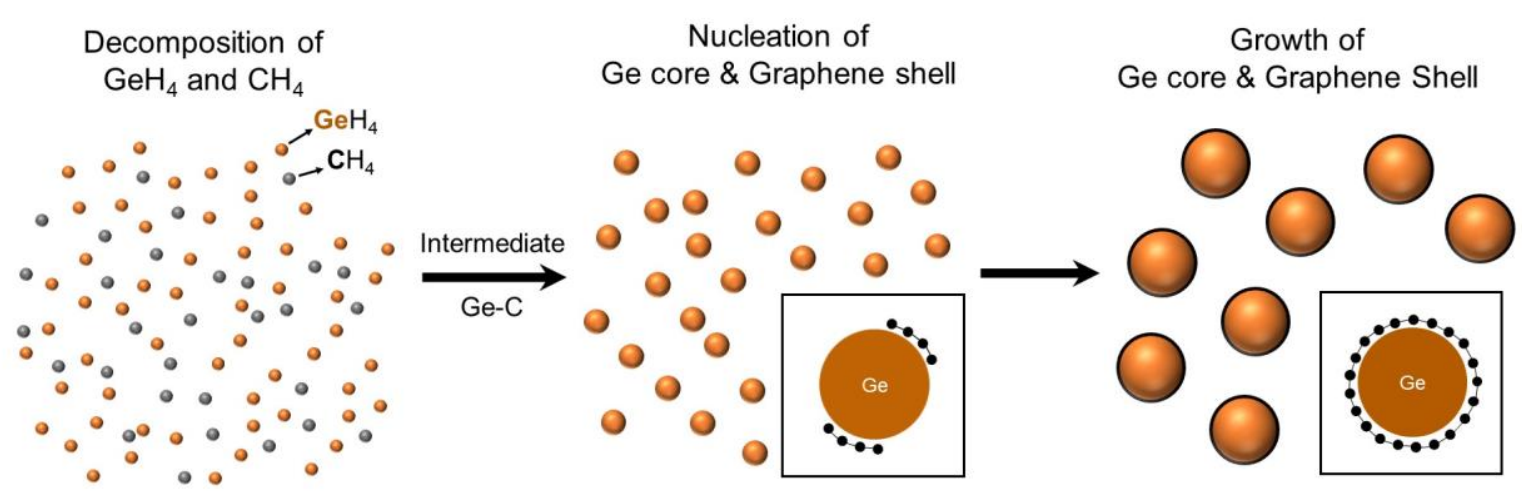

Fig. 4. Schematic of the growth mechanism of the Ge@G NCs.

On the basis of the experimental results, we are able to propose a possible growth mechanism of the Ge@G NCs, as shown in Fig. 4. The overall process follows three growth stages: (1) Thermal decomposition of germane and methane; (2) nucleation of the Ge core and graphene shell; and (3) agglomeration of the Ge core and the growth of the graphene shell. In particular, we believe that methane plays a critical role in the growth of the graphene shell, as well as in the determination of the nucleation density and diameter of the $\mathrm{Ge} @ \mathrm{G}$ NCs, similar to a surfactant in the liquid-phase growth of the NCs [33, 34].

At the initial stage of the Ge@G NCs growth, methane can affect the nucleation density. When the growth temperature is higher than $800{ }^{\circ} \mathrm{C}$, the gaseous mixture of methane and germane is completely decomposed and produces labile $\mathrm{Ge}-\mathrm{C}$ molecules. As $\mathrm{Ge}-\mathrm{C}$ is thermodynamically unstable at the growth temperature $[35,36]$, they begin to agglomerate with each other, and then produce Ge clusters as a seed for the Ge core. If the growth temperature is decreased lower than $800{ }^{\circ} \mathrm{C}$, which is the critical decomposition temperature of methane, the density of the Ge@G NCs is also significantly reduced (see Fig. S4) [37].

In typical pyrolysis reactions, the size of the NCs is controlled by the residence time of the precursors under the same pressure and growth temperature $[16,38]$. Here, in contrast, the average diameter of the NCs is determined by the partial pressure of methane, which is related to the growth rate of the graphene shell, as shown in Fig. 2 and Fig. S2. Because Ge 
shows a good catalytic behavior for $s p^{2}$-hybridization, both the Ge core and graphene shell are synthesized simultaneously through the entire growth process. Further, although the sample is still passing through the hot-region of the quartz tube, the growth of the Ge@G NCs can be stopped when the graphene shell completely covers the surface of the NCs, because the graphene shell effectively prohibits further growth of the Ge core [39]. If the flow rate of methane is decreased to as small as 1 standard cubic centimeter per minute, larger, irregularly shaped NCs are formed with a partially covered graphene shell, as shown in Fig. S5.

\subsection{Thermal and electrical conductivity of the Ge@GNCs}
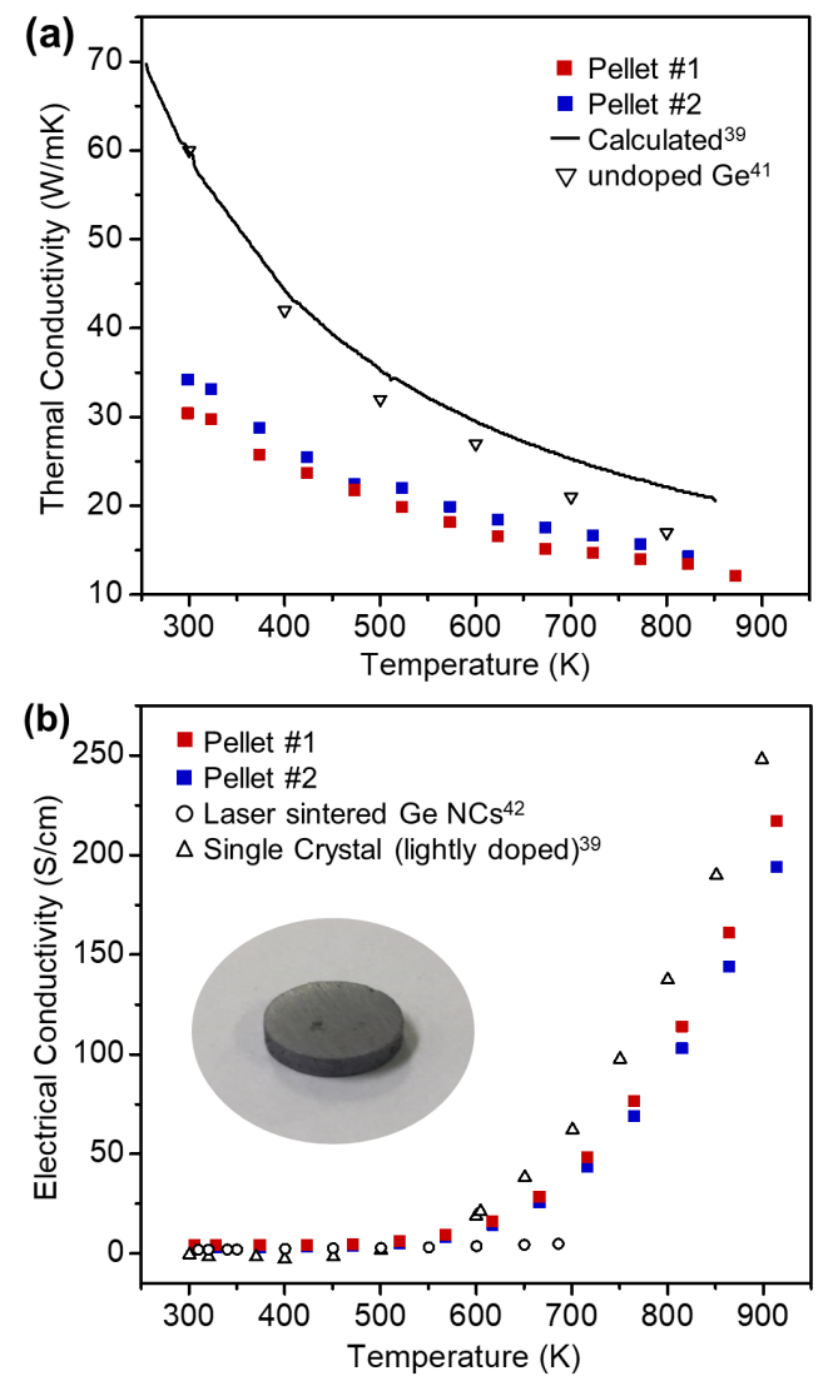
Fig. 5. (a) Thermal and (b) electric conductivity of the Ge@G NCs pellet as a function of temperature. Inset of (b) shows a photograph of the Ge@G NCs pellet.

To further investigate the merits of the graphene shell for device applications, temperature dependence of the thermal and electric conductivity measurements was evaluated. Two sintered pellets of the $\mathrm{Ge} @ \mathrm{G}$ NCs were prepared by a spark plasma sintering method (Inset of Fig. 5 (b)). Although the value of thermal conductivity of the Ge@G NCs pellets can be expected to be increased due to the high thermal conductivity of graphene, both pellets show significant decrease in thermal conductivity compared to the theoretical and measured conductivity values of un-doped single crystal Ge substrate. (Fig. 5 (a)) [40-42]. There are various factors that can reduce the overall thermal conductivity of the Ge@G NC pellet. In general, the lattice thermal conductivity is directly related to the mean free path of phonon, and large density of grain boundaries generates phonon-grain boundary scattering [43-47]. Therefore, the thermal conductivity decreases sharply when the size of grain is close to the nanometer scale $(<100 \mathrm{~nm})$. Based on the theoretical studies, we can predict the thermal conductivity of individual nanocrystalline graphene shell. Since the estimated domain size of the graphene is approximately $11.31 \mathrm{~nm}$, the calculated thermal conductivity is close to the $25 \mathrm{~W} / \mathrm{mK}$, two orders of magnitude smaller than that of the freestanding graphene [44, 48]. The phonon scattering also occurs at the interface between Ge and graphene because of strong flexural phonon scattering (ZA mode or our-of-plane transverse acoustic mode), causing dramatic reduction of the thermal conductivity $[43,48]$. Above all, as the heat moves in the form of the flux, the thermal conductivity of the Ge@G NCs cannot be calculated by simply combining the thermal conductivity of Ge and graphene $[49,50]$. It should be calculated considering the cross-sectional area $(A)$ portion of each other. From the HR-TEM image of Ge@G NCs in Fig. 2(d), $A_{\text {shell }}$ is less than 3\% of the total cross-sectional area. 
In contrast, it was found that the electrical conductivity of the graphene incorporated pellets (pellets \#1 and \#2) are comparable to that of lightly-doped single crystalline Ge wafer (Ga doped, $5.7 \times 10^{16}$ ), while being higher than that of a laser-sintered un-doped Ge NCs film $[40,51]$.
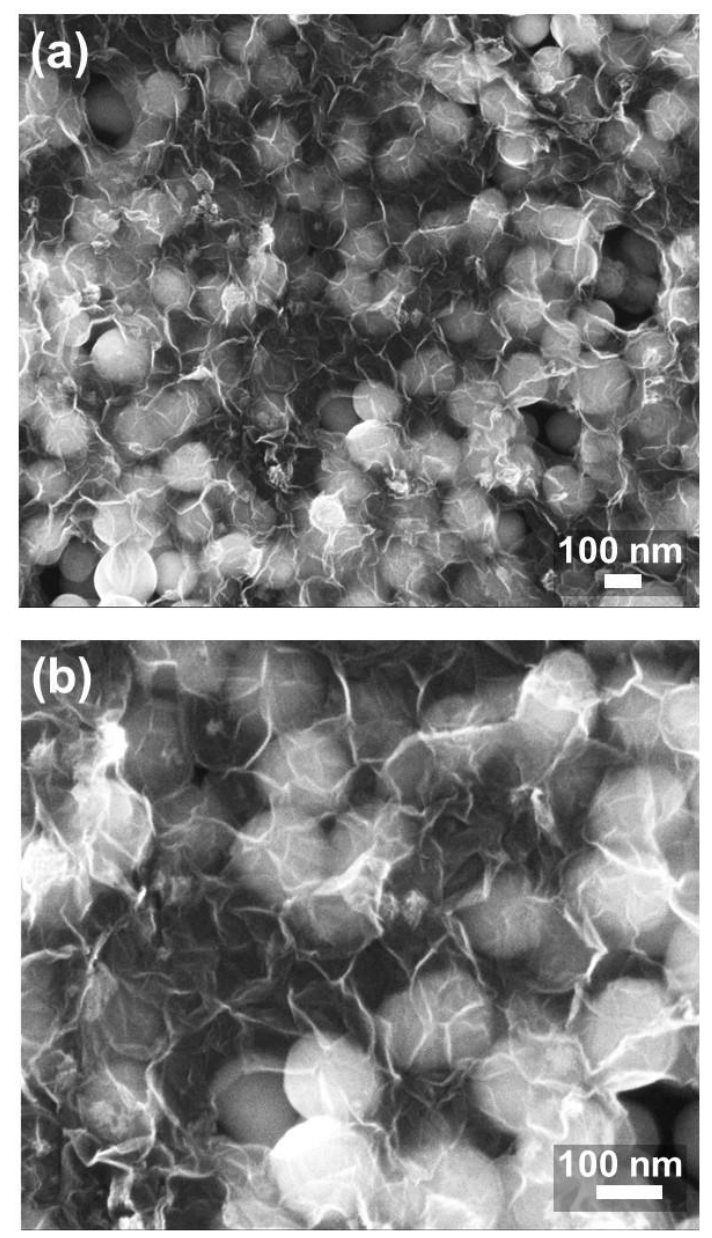

Fig. 6 (a) and (b) SEM images of bubble-wrap structure of graphene after wet-chemical etching process.The Ge@G NCs film is prepared by annealing of the Ge@G NCs at $500^{\circ} \mathrm{C}$ for $1 \mathrm{~h}$. And then we dipped it into a mixed solution containing $\mathrm{H}_{2} \mathrm{O} / \mathrm{H}_{2} \mathrm{O}_{2}$ (6:1) for $30 \mathrm{~min}$.

These results can be elucidated by the interconnected 3D network of graphene in the Ge@G NCs pellets (Fig. 6). Although un-doped Ge shows a low intrinsic charge carrier density at the lower temperature region, the conductive bubble-wrap structure of graphene helps to increase the overall electronic conductivity of the sintered pellets (See Table S2) [11, 50, 52-56]. Moreover, when the temperature is higher than $600 \mathrm{~K}$, it can work as a charge 
collector for the thermally activated excess charge carriers. Thus, the results confirm that the presence of the graphene shell is useful for satisfying the conditions that improve the performance of a thermoelectric device based on nanomaterials, which should be lowered thermal conductivity, without any reduction of electric conductivity. We expect that adding various elements into the Ge core will further enhance the thermoelectric performance [40, $51,57]$.

\section{Conclusion}

In conclusion, we introduced a facile, one-pot, and scalable process for the growth of graphene-coated Ge NCs using a conventional CVD method. Unlike other typical gas-phase growth methods, we could control the diameter of the Ge@G NCs in the range of $\sim 5 \mathrm{~nm}$ to $\sim 180 \mathrm{~nm}$ by tuning the gas ratio of methane and germane. Spectroscopic and microscopic analysis confirms that the Ge core of the $\mathrm{NC}$ is single crystalline, and is completely covered by a multi-layered graphene shell. We believe that this growth approach will provide a facile route for the scalable growth of uniform semiconductor NCs, such as Si and SiGe alloys.

\section{Acknowledgement}

This work was supported by the Presidential Postdoctoral Fellowship Program of the Ministry of Education, through the NRF (2014R1A6A3A04058169).

\section{Appendix A: Supplementary data}

Supplementary data associated with this article can be found in the online version at http://dx.doi.org/ 


\section{References}

[1] K. Kim, J.Y. Choi, T. Kim, S.H. Cho, H.J. Chung, A role for graphene in silicon-based semiconductor devices, Nature, 479 (2011) 338-344.

[2] X. Wang, Z. Cheng, K. Xu, H.K. Tsang, J.B. Xu, High-responsivity graphene/siliconheterostructure waveguide photodetectors, Nat. Photon., 7 (2013) 888-891.

[3] N. Youngblood, Y. Anugrah, R. Ma, S.J. Koester, M. Li, Multifunctional graphene optical modulator and photodetector integrated on silicon waveguides, Nano Lett., 14 (2014) 2741 2746

[4] H. Yang, J. Heo, S. Park, H.J. Song, D.H. Seo, K.E. Byun, P. Kim, I. Yoo, H.J. Chung, K. Kim, Graphene barristor, a triode device with a gate-controlled Schottky barrier, Science, 336 (2012) 1140-1143.

[5] W. Yao, Y. Cui, L. Zhan, F. Chen, Y. Zhang, Y. Wang, S. Y., Two-dimensional sandwichlike Ag coated silicon-graphene-silicon nanostructures for superior lithium storage, Appl. Surf. Sci., 425 (2017) 614-621.

[6] J. Yu, J. Yang, X. Feng, H. Jia, J. Wang, W. Lu, Uniform carbon coating on silicon nanoparticles by dynamic CVD process for electrochemical lithium storage, Ind. Eng. Chem. Res., 53 (2014) 12697-12704.

[7] Y. Li, K. Yan, H.-W. Lee, Z. Lu, N. Liu, Y. Cui, Growth of conformal graphene cages on micrometre-sized silicon particles as stable battery anodes, Nat. Energy., 1 (2016) 15029.

[8] S. Lee, J. Hong, J.H. Koo, H. Lee, S. Lee, T. Choi, H. Jung, B. Koo, J. Park, H. Kim, Y.W. Kim, T. Lee, Synthesis of few-layered graphene nanoballs with copper cores using solid carbon source, ACS Appl. Mater. Interfaces, 5 (2013) 2432-2437.

[9] J. Zhu, Y. Li, S. Kang, X.L. Wei, P.K. Shen, One-step synthesis of $\mathrm{Ni}_{3} \mathrm{~S}_{2}$ nanoparticles wrapped with in situ generated nitrogen-self-doped graphene sheets with highly improved electrochemical properties in Li-ion batteries, J. Mater. Chem. A, 2 (2014) 3142-3147. 
[10] S.M. Yoon, W.M. Choi, H. Baik, H.J. Shin, I. Song, M.S. Kwon, J.J. Bae, H. Kim, Y.H. Lee, J.Y. Choi, Synthesis of multilayer graphene balls by carbon segregation from nickel nanoparticles, ACS Nano, 6 (2012) 6803-6811.

[11] D.J. Xue, S. Xin, Y. Yan, K.C. Jiang, Y.X. Yin, Y.G. Guo, L.J. Wan, Improving the electrode performance of Ge through Ge@C core-shell nanoparticles and graphene networks, J. Am. Chem. Soc., 134 (2012) 2512-2515.

[12] Y. Ma, Z. Hu, L. Yu, Y. Hu, B. Yue, X. Wang, Y. Chen, Y. Lu, Y. Liu, J. Hu, Chemical functionalization of magnetic carbon-encapsulated nanoparticles based on acid oxidation, J. Phys. Chem. B, 110 (2006) 20118-20122.

[13] M.A. Zalich, V.V. Baranauskas, J.S. Riffle, M. Saunders, T.G. St Pierre, Structural and magnetic properties of oxidatively stable cobalt nanoparticles encapsulated in graphite shells, Chem. Mater., 18 (2006) 2648-2655.

[14] S. Wang, X. Huang, Y. He, H. Huang, Y. Wu, L. Hou, X. Liu, T. Yang, J. Zou, B. Huang, Synthesis, growth mechanism and thermal stability of copper nanoparticles encapsulated by multi-layer graphene, Carbon, 50 (2012) 2119-2125.

[15] S.K. Sengar, B.R. Mehta, R. Kumar, V. Singh, In-flight gas phase growth of metal/multi layer graphene core shell nanoparticles with controllable sizes, Sci. Rep., 3 (2013) 2814.

[16] R. Gresback, Z. Holman, U. Kortshagen, Nonthermal plasma synthesis of sizecontrolled, monodisperse, freestanding germanium nanocrystals, Appl. Phys. Lett., 91 (2007) 093119.

[17] Z.C. Holman, C.Y. Liu, U.R. Kortshagen, Germanium and silicon nanocrystal thin-film field-effect transistors from solution, Nano Lett., 10 (2010) 2661-2666.

[18] M.H. Park, K. Kim, J. Kim, J. Cho, Flexible dimensional control of high-capacity LiIon-battery anodes: from 0D hollow to 3D porous germanium nanoparticle assemblies, Adv. Mater., 22 (2010) 415-418. 
[19] L.M. Wheeler, A.W. Nichols, B.D. Chernomordik, N.C. Anderson, M.C. Beard, N.R. Neale, All-Inorganic Germanium Nanocrystal Films by Cationic Ligand Exchange, Nano Lett., 16 (2016) 1949-1954.

[20] J.H. Lee, E.K. Lee, W.J. Joo, Y. Jang, B.S. Kim, J.Y. Lim, S.H. Choi, S.J. Ahn, J.R. Ahn, M.H. Park, C.W. Yang, B.L. Choi, S.W. Hwang, D. Whang, Wafer-scale growth of singlecrystal monolayer graphene on reusable hydrogen-terminated germanium, Science, 344 (2014) 286-289.

[21] G. Wang, M. Zhang, Y. Zhu, G. Ding, D. Jiang, Q. Guo, S. Liu, X. Xie, P.K. Chu, Z. Di, X. Wang, Direct growth of graphene film on germanium substrate, Sci. Rep., 3 (2013) 2465. [22] T. Uchino, K.N. Bourdakos, C.H. De Groot, P. Ashburn, M.E. Kiziroglou, G.D. Dilliway, D.C. Smith, Metal catalyst-free low-temperature carbon nanotube growth on SiGe islands, Appl. Phys. Lett., 86 (2005) 233110.

[23] A.S. Okhotin, A.S. Pushkarskij, V.V. Gorbachev, Thermophysical properties of semiconductor, Atom Publ. House, Moscow, 1972.

[24] J.H. Parker, D.W. Feldman, M. Ashkin, Raman scattering by silicon and germanium, Phys. Rev., 155 (1967) 712-714.

[25] A.C. Ferrari, J.C. Meyer, V. Scardaci, C. Casiraghi, M. Lazzeri, F. Mauri, S. Piscanec, D. Jiang, K.S. Novoselov, S. Roth, Raman spectrum of graphene and graphene layers, Phys. Rev. Lett., 97 (2006) 187401.

[26] C. Casiraghi, A. Hartschuh, H. Qian, S. Piscanec, C. Georgi, A. Fasoli, K.S. Novoselov, D.M. Basko, A.C. Ferrari, Raman Spectroscopy of Graphene Edges, Nano Lett., 9 (2009) $1433-1441$.

[27] L.G. Caņado, K. Takai, T. Enoki, M. Endo, Y.A. Kim, H. Mizusaki, A. Jorio, L.N. Coelho, R. Magalhães-Paniago, M.A. Pimenta, General equation for the determination of the crystallite size la of nanographite by Raman spectroscopy, Appl Phys Lett, 88 (2006). 
[28] J.Y. Jao, S. Han, L.S. Chang, Y.C. Chen, C.L. Chang, H.C. Shih, Formation and characterization of DLC:Cr:Cu multi-layers coating using cathodic arc evaporation, Diamond Relat. Mater., 18 (2009) 368-373.

[29] J. Vilcarromero, F.C. Marques, XPS study of the chemical bonding in hydrogenated amorphous germanium-carbon alloys, Appl. Phys. A: Mater. Sci. Process., 70 (2000) 581585.

[30] J.H. Lee, S.H. Choi, S.P. Patole, Y. Jang, K. Heo, W.J. Joo, J.B. Yoo, S.W. Hwang, D. Whang, Reliability enhancement of germanium nanowires using graphene as a protective layer: Aspect of thermal stability, ACS Appl. Mater. Interfaces, 6 (2014) 5069-5074.

[31] S. Chen, L. Brown, M. Levendorf, W. Cai, S.Y. Ju, J. Edgeworth, X. Li, C.W. Magnuson, A. Velamakanni, R.D. Piner, J. Kang, J. Park, R.S. Ruoff, Oxidation resistance of graphene-coated $\mathrm{Cu}$ and $\mathrm{Cu} / \mathrm{Ni}$ alloy, ACS Nano, 5 (2011) 1321-1327.

[32] R.I. Scace, G.A. Slack, Solubility of carbon in silicon and germanium, J. Chem. Phys., 30 (1959) 1551-1555.

[33] Y. Bao, W. An, C. Heath Turner, K.M. Krishnan, The critical role of surfactants in the growth of cobalt nanoparticles, Langmuir, 26 (2010) 478-483.

[34] N.T.K. Thanh, N. Maclean, S. Mahiddine, Mechanisms of nucleation and growth of nanoparticles in solution, Chem. Rev., 114 (2014) 7610-7630.

[35] J.L. Bills, F.A. Cotton, The enthalpy of formation of tetraethylgermane and the germanium-carbon bond energy, J. Phys. Chem., 68 (1964) 806-810.

[36] J.H. Lee, M.S. Kim, J.Y. Lim, S.H. Jung, S.G. Kang, H.J. Shin, J.Y. Choi, S.W. Hwang, D. Whang, CMOS-compatible catalytic growth of graphene on a silicon dioxide substrate, Appl. Phys. Lett., 109 (2016) 053102.

[37] T.V. Choudhary, D.W. Goodman, Methane decomposition: production of hydrogen and carbon filaments, Catalysis, 19 (2006) 164-183. 
[38] M. Gröschel, R. Körmer, M. Walther, G. Leugering, W. Peukert, Process control strategies for the gas phase synthesis of silicon nanoparticles, Chem. Eng. Sci., 73 (2012) 181-194.

[39] B.S. Kim, M.J. Kim, J.C. Lee, S.W. Hwang, B.L. Choi, E.K. Lee, D. Whang, Control of lateral dimension in metal-catalyzed germanium nanowire growth: Usage of carbon sheath, Nano Lett., 12 (2012) 4007-4012.

[40] Y. Ohishi, S. Takarada, Y. Aikebaier, H. Muta, K. Kurosaki, S. Yamanaka, Y. Miyazaki, N. Uchida, T. Tada, Thermoelectric properties of gallium-doped p-type germanium, Jpa. J. Appl. Phys., 55 (2016) 051301.

[41] M. Ohtaki, K. Araki, K. Yamamoto, High thermoelectric performance of dually doped ZnO ceramics, J. Electron. Mater., 38 (2009) 1234-1238.

[42] C.J. Glassbrenner, G.A. Slack, Thermal Conductivity of Silicon and Germanium from $3^{\circ} \mathrm{K}$ to the Melting Point, Phys. Rev., 134 (1964) A1058.

[43] A.Y. Serov, Z.Y. Ong, E. Pop, Effect of grain boundaries on thermal transport in graphene, Appl Phys Lett, 102 (2013).

[44] K.R. Hahn, C. Melis, L. Colombo, Thermal transport in nanocrystalline graphene investigated by approach-to-equilibrium molecular dynamics simulations, Carbon, 96 (2016) $429-438$

[45] C.M. Bhandari, D.M. Rowe, Boundary scattering of phonons, Journal of Physics C: Solid State Physics, 11 (1978) 1787-1794.

[46] N. Petermann, N. Stein, G. Schierning, R. Theissmann, B. Stoib, M.S. Brandt, C. Hecht, C. Schulz, H. Wiggers, Plasma synthesis of nanostructures for improved thermoelectric properties, J. Phys. D: Appl. Phys., 44 (2011) 174034.

[47] Z. Wang, J.E. Alaniz, W. Jang, J.E. Garay, C. Dames, Thermal conductivity of nanocrystalline silicon: Importance of grain size and frequency-dependent mean free paths, 
Nano Lett., 11 (2011) 2206-2213.

[48] J.H. Seol, I. Jo, A.L. Moore, L. Lindsay, Z.H. Aitken, M.T. Pettes, X. Li, Z. Yao, R. Huang, D. Broido, N. Mingo, R.S. Ruoff, L. Shi, Two-dimensional phonon transport in supported graphene, Science, 328 (2010) 213-216.

[49] M. Hu, X. Zhang, K.P. Giapis, D. Poulikakos, Thermal conductivity reduction in coreshell nanowires, Physical Review B - Condensed Matter and Materials Physics, 84 (2011).

[50] J.W. Lee, E.K. Lee, B.S. Kim, J.H. Lee, H.G. Kim, H.S. Jang, S.W. Hwang, B.L. Choi, D. Whang, Thermoelectric Properties of Nanowires with a Graphitic Shell, ChemSusChem, 8 (2015) 2372-2377.

[51] B. Stoib, T. Langmann, N. Petermann, S. Matich, M. Sachsenhauser, H. Wiggers, M. Stutzmann, M.S. Brandt, Morphology, thermoelectric properties and wet-chemical doping of laser-sintered germanium nanoparticles, Phys. Status Solidi A, 210 (2013) 153-160.

[52] T.K. Gupta, W.G. Carlson, A grain-boundary defect model for instability/stability of a $\mathrm{ZnO}$ varistor, Journal of Materials Science, 20 (1985) 3487-3500.

[53] W.H. Nam, Y.S. Lim, S.M. Choi, W.S. Seo, J.Y. Lee, High-temperature charge transport and thermoelectric properties of a degenerately Al-doped $\mathrm{ZnO}$ nanocomposite, J. Mater. Chem., 22 (2012) 14633-14638.

[54] W.H. Nam, B.B. Kim, S.G. Seo, Y.S. Lim, J.Y. Kim, W.S. Seo, W.K. Choi, H.H. Park, J.Y. Lee, Structurally nanocrystalline-electrically single crystalline zno-reduced graphene oxide composites, Nano Lett., 14 (2014) 5104-5109.

[55] Y. Sun, X. Hu, W. Luo, Y. Huang, Self-assembled hierarchical $\mathrm{MoO}<\mathrm{inf}>2</$ inf $>/$ graphene nanoarchitectures and their application as a high-performance anode material for lithium-ion batteries, ACS Nano, 5 (2011) 7100-7107.

[56] S. Yang, X. Feng, S. Ivanovici, K. Müllen, Fabrication of graphene-encapsulated oxide nanoparticles: Towards high-performance anode materials for lithium storage, Angewandte 
Chemie - International Edition, 49 (2010) 8408-8411.

[57] J.A. Martinez, P.P. Provencio, S.T. Picraux, J.P. Sullivan, B.S. Swartzentruber, Enhanced thermoelectric figure of merit in SiGe alloy nanowires by boundary and hole-phonon scattering, J. of Appl. Phys., 110 (2011) 074317. 